\title{
CBMA [Chartae Burgundiae Medii Aevi] - V. Actes cisterciens et prémontrés
}

Dijon, UMR 5594 Artehis, 28 janvier 2011

Eliana Magnani et Marie-José Gasse-Grandjean

\section{(2) OpenEdition}

\section{Journals}

Édition électronique

URL : https://journals.openedition.org/cem/11991

DOI : 10.4000/cem. 11991

ISSN : 1954-3093

Éditeur

Centre d'études médiévales Saint-Germain d'Auxerre

Édition imprimée

Pagination : 00

ISSN : 1623-5770

\section{Référence électronique}

Eliana Magnani et Marie-José Gasse-Grandjean, «CBMA [Chartae Burgundiae Medii Aevi] - V. Actes cisterciens et prémontrés », Bulletin du centre d'études médiévales d'Auxerre | BUCEMA [En ligne], 15 |

2011, mis en ligne le 26 mars 2013, consulté le 22 septembre 2022. URL : http://

journals.openedition.org/cem/11991; DOI : https://doi.org/10.4000/cem.11991

Ce document a été généré automatiquement le 22 septembre 2022.

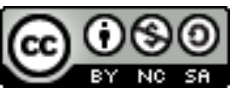

Creative Commons - Attribution - Pas d'Utilisation Commerciale - Partage dans les Mêmes Conditions 4.0 International - CC BY-NC-SA 4.0

https://creativecommons.org/licenses/by-nc-sa/4.0/ 


\title{
CBMA [Chartae Burgundiae Medii Aevi] - V. Actes cisterciens et prémontrés
}

Dijon, UMR 5594 Artehis, 28 janvier 2011

\author{
Eliana Magnani et Marie-José Gasse-Grandjean
}

1 C'est à l'aune de la double ambition du programme des CBMA - le libre accès à une masse de documents numérisés propre à modifier nos méthodes de recherche et le regroupement de chercheurs intéressés à ce nouveau développement - que sa cinquième journée d'études s'est déroulée. L'enrichissement du portail documentaire, les évolutions techniques et le web semantic ont constitué le premier volet des travaux, suivis des études sur la documentation comptable et diplomatique bourguignonne médiévale.

\section{Les nouveaux outils web}

Depuis l'été 2010, comme l'a exposé Marie-José Gasse-Grandjean, les 11400 actes diplomatiques numérisés par les CBMA son interrogeables avec l'application de fouille de textes Philologic: http://www.artehis-cbma.eu. Doté d'une interface d'interrogation adaptée et d'une aide à l'utilisateur simplifiée, traduites en français, il s'agit du premier ensemble de milliers de textes diplomatiques qui peut désormais être soumis à une enquête poussée. Grâce à l'aide d'Alain Guerreau et au travail de Nicolas Perreaux doctorant de l'université de Bourgogne, Artehis -, cette nouvelle version, accessible à partir d'un serveur dédié ${ }^{1}$, hébergé dans les locaux d'Artehis, succède à celle que Mark Olsen a accueillie avec bienveillance pendant quatre ans sur un serveur de l'université de Chicago. Nous tenons à les saluer ici pour leur inestimable collaboration. Avec ces améliorations, nous espérons stimuler l'utilisation de cet instrument et nous consacrerons notre prochaine journée d'études, le 27 janvier 2012, aux travaux émanant de ces enquêtes (fig. 1). 


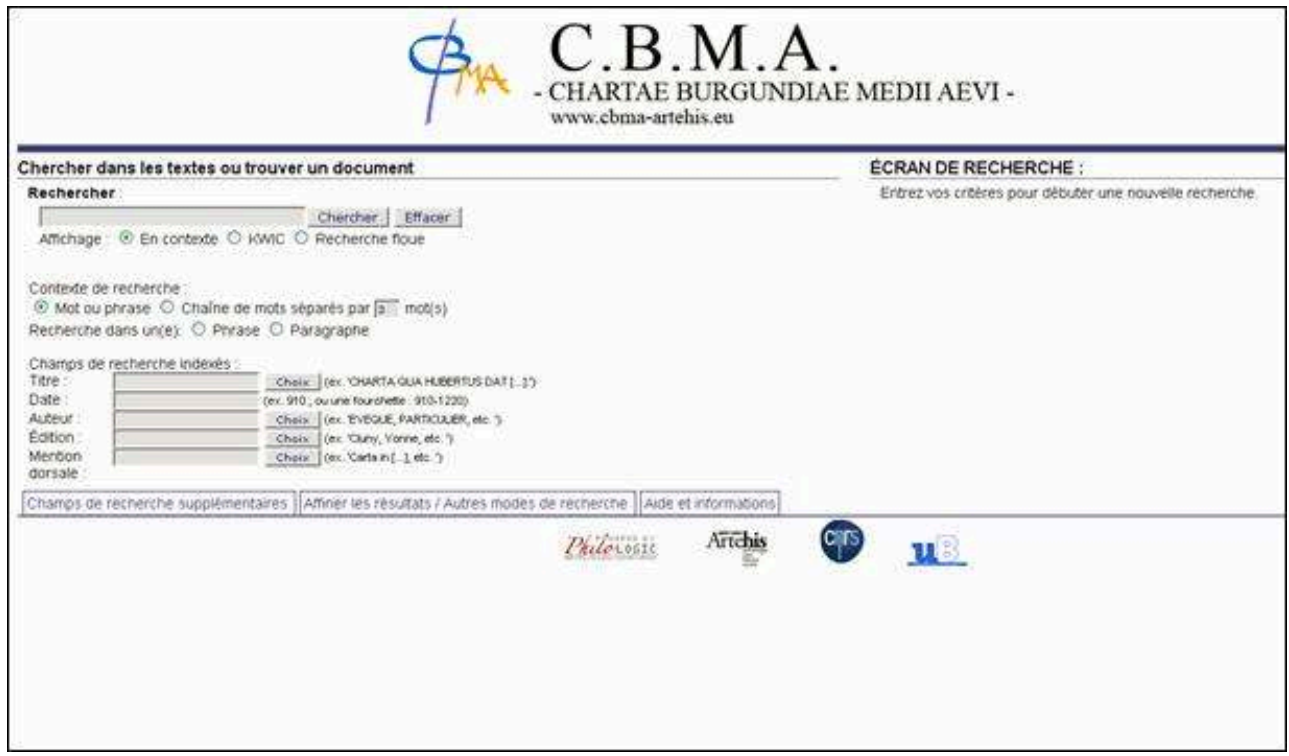

Fig. 1 - Serveur CBMA hébergé dans les locaux d'Artehis et permettant d'interroger les chartes bourguignonnes sous Philologic [http://www.cbma-artehis.eu]

3 Une approche spatialisée des corpus, réunis par le projet CBMA, est désormais également possible à travers l'application cartographique développée par Ludovic Granjon - Maison des sciences de l'Homme de Dijon ${ }^{2}$ : http://phalombe.ubourgogne.fr/cbma/. Elle propose diverses options permettant d'affiner la recherche dont le tri des établissements ecclésiastiques par patronage -, et l'affichage des résultats, par exemple une carte dynamique des diocèses et des légendes ${ }^{3}$.

4 En vue de donner une nouvelle visibilité aux CBMA, le blog Laetus diaconus a été créé en octobre 2010 [http://laetusdiaconus.hypotheses.org/], à l'intérieur d'un espace d'échanges très ouvert, élargi aux pratiques de l'écrit et du visuel au Moyen Âge. Cet outil a été confié aux doctorants travaillant sur la documentation bourguignonne afin de nouer des contacts et de disposer d'un espace de discussion étendu à un contexte SHS, qui est celui de la plate-forme «Hypothèses» du CLEO. L'annonce d'une manifestation, une note de lecture ou un compte rendu de rencontre, le signalement d'un site ou d'un document d'archive intéressant, la formulation d'une réflexion sont autant de thèmes qui peuvent y être publiés. Nous vous encourageons tous à consulter ce blog et à y participer (fig. 2). 


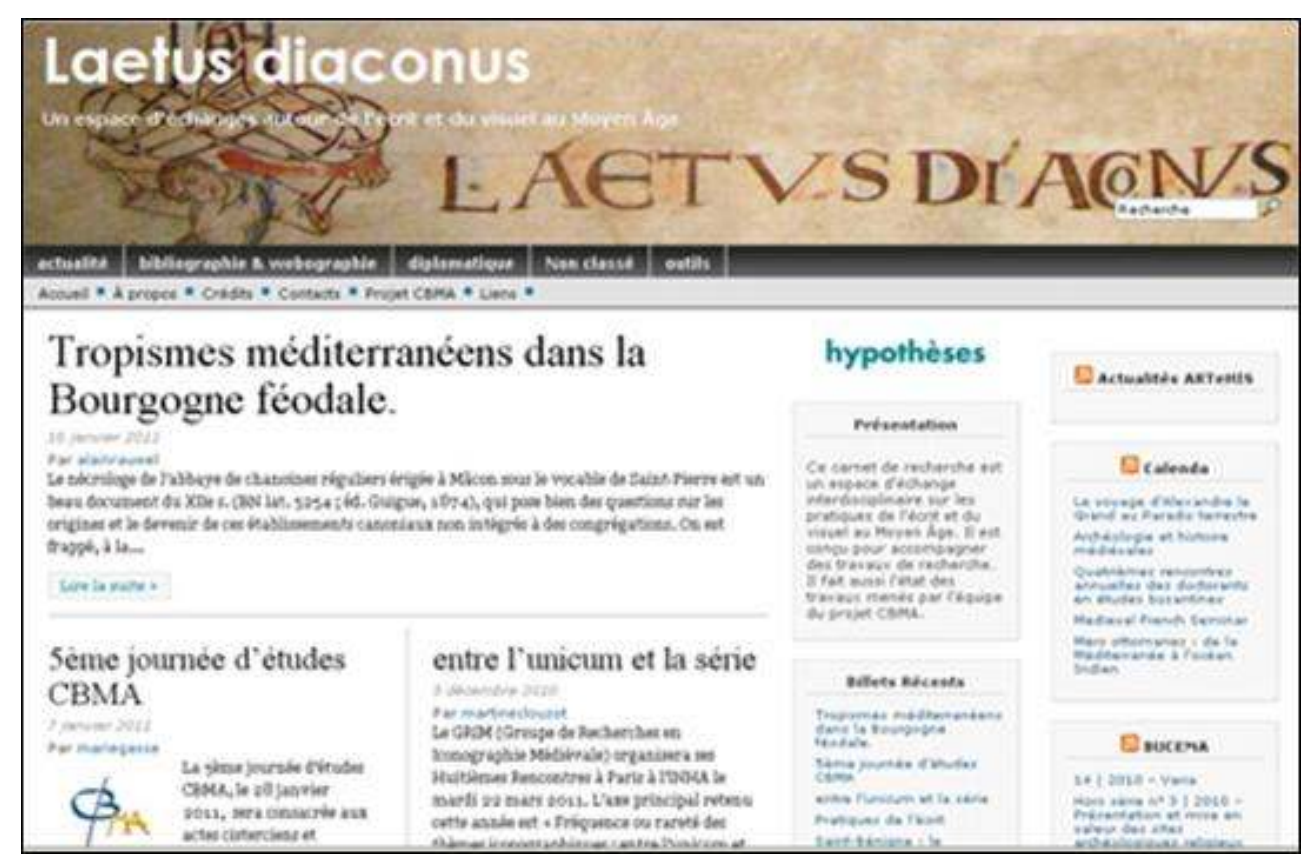

Fig. 2 - Blog Laetus diaconus [http://laetusdiaconus.hypotheses.org/]

5 Parallèlement à ces innovations, la documentation texte et image du portail des CBMA a continué d'être enrichie [http://www.artehis-cnrs.fr/CBMA-Chartae-BurgundiaeMedii-Aevi]. Trois nouveaux ensembles diplomatiques ont été enregistrés, traités et mis à disposition. Les nouveautés sont désormais signalées par un petit gif animé «new » : le cartulaire de la ville d'Arbois (83 actes) ${ }^{4}$, les preuves de l'histoire de Gigny (82 actes) ${ }^{5}$ et le cartulaire de Marcigny-sur-Loire (252 actes) ${ }^{6}$. Le long travail de documentation de la base de données s'est poursuivi en 2010, grâce au travail de deux étudiants contractuels, Nicolas Perreaux (université de Bourgogne) et Benjamin Lefèvre (université de Tours) : 1000 actes supplémentaires ont été analysés. Au total, près de la moitié des actes diplomatiques enregistrés sont aujourd'hui renseignés. Parmi les codices à "feuilleter » en mode image, trois cartulaires ont été ajoutés à la série des manuscrits numérisés par les archives départementales de la Côte-d'Or, deux cartulaires de Cîteaux du XIII ${ }^{\mathrm{e}}$ siècle ${ }^{7}$, le tome VI du grand cartulaire de Jean de Cirey du $\mathrm{XV}^{\mathrm{e}}$ siècle ${ }^{8}$ et le cartulaire du prieuré Saint-Vivant de Vergy ${ }^{9}$. Des fonctions de téléchargement, d'impression, un zoom efficace et une grande facilité de déplacement dans le document définissent un mode d'accès souple et de qualité, particulièrement apprécié par les chercheurs étrangers.

Soucieuse du partage et de l'échange d'expériences, l'équipe des CBMA a invité le chercheur suédois Johan Ahlfeldt à présenter son projet Regnum Francorum Online (RFO, http://www.francia.ahlfeldt.se// ${ }^{10}$. Partant du constat que la documentation disponible sur internet ne cesse d'augmenter et que toutes les sciences sont intéressées par la recherche cartographique, Johan Ahlfeldt a élaboré une application cartographique très étoffée, permettant de lier et de visualiser divers types de documents du haut Moyen Âge - des textes littéraires et diplomatiques, des monnaies, des atlas... - à une série d'événements. Cette mise en contexte, à l'échelle de l'Europe, pour la période 614-840, lui permet de présenter différentes strates thématisées : chartes de Charles le Mauvais, itinéraire de Charlemagne, possessions de l'abbaye de Saint-Denis, fisc du royaume franc... Une aide à la consultation, un calendrier médiéval et la liste des 
pagi figurent parmi les nombreux éléments auxiliaires disponibles pour les utilisateurs. Parmi les dernières avancées du RFO, des liens vers GoogleMap se révèlent parfois spectaculaires quand ils laissent découvrir les vues aériennes des traces de substructions médiévales. Le prolongement chronologique jusqu'à l'année 950, la possibilité de sélection/désélection de points, un choix plus étendu de cartes thématiques, ainsi qu'une aide en français, font partie des perfectionnements envisagés prochainement. Cette collecte, tout à la fois compilation et organisation de la documentation online, pose toute une série de problèmes techniques et conceptuels, allant de la toponymie ancienne au besoin urgent d'identifiants web uniques et stables. Le blog Early Medieval Mapping (http://early-medieval-gis.blogspot.com/) est ainsi le lieu ouvert aux questions et aux discussions au sujet des méthodes et des techniques du RFO (fig. 3).

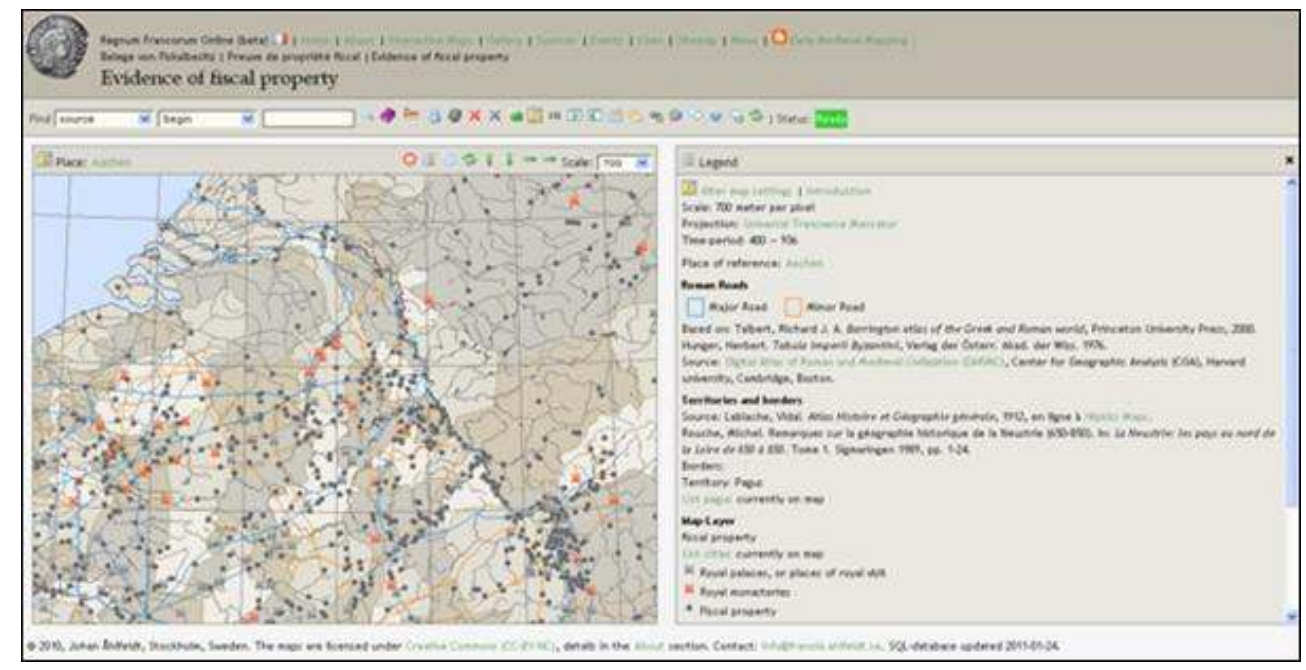

Fig. 3 - Regnum Francorum Online [http://www.francia.ahlfeldt.se/]

\section{Pratiques de l'écrit}

7 Même si la diplomatique constitue le centre du programme des CBMA, nous l'orientons vers une compréhension élargie et sur la longue durée, de la génétique du document, des pratiques de l'écrit et de ses procédés graphiques. Le volume en préparation Productions, remplois, mises en registre : la pratique sociale de l'écrit à travers la documentation médiévale bourguignonne, regroupant les communications du colloque tenu à Auxerre en 2009, ainsi que des contributions complémentaires, devrait refléter l'état actuel de la recherche dans ce domaine ${ }^{11}$. Nous avons également attiré l'attention sur les fragments de livres de chant médiévaux conservés dans les reliures de manuscrits et les couvertures des liasses des archives monastiques et nous accompagnons la réalisation de l'inventaire et l'étude de ces fragments, travail en cours mené par Eduardo H. Aubert (doctorant à l'EHESS) ${ }^{12}$.

8 C'est aussi dans le sens d'une approche globale des pratiques de l'écrit que nous nous sommes associés à l'équipe dirigée par Patrice Beck - IRHiS, université Charles de Gaulle Lille 3 -, travaillant sur les comptabilités ${ }^{13}$, pour l'organisation de la table ronde Le vocabulaire et la rhétorique des comptabilités médiévales. Modèles, innovations, formalisation - Dijon/Besançon, 30 septembre- $1^{\mathrm{er}}$ octobre $2010^{14}$ - et avons invité les chercheurs étudiants les comptabilités bourguignonnes à faire état de leurs recherches lors de nos 
journées d'études. Cette année, Sylvie Bépoix a présenté une série d'observations à partir de l'étude comparée des comptabilités domaniales des $\mathrm{XIV}^{\mathrm{e}}$ et $\mathrm{XV}^{\mathrm{e}}$ siècles du comté et du duché de Bourgogne - châtellenie comtale de Saint-Aubin ou Bracon, châtellenies ducales de Semur-en-Auxois et de Vergy. Les données mises en perspective sont issues de ses travaux ainsi que de ceux de Matthieu Leguil et de Fabienne Couvel, qui terminent actuellement leur thèse ${ }^{15}$. Sur la base de l'ordonnance de Philippe le Hardi de 1386, qui fixe par écrit des pratiques antérieures et de l'analyse de la matérialité et de l'organisation des comptes - support, mise en page, organisation des cahiers, plan...--, on constate la convergence entre les pratiques du duché et du comté, lors de la mise en place de ces instruments de gestion, de mise en valeur et de mémoire du domaine, conçus pour le service du prince. Les acteurs du compte, receveurs et gens de la chambre des comptes, sont soumis à des règles strictes et à des procédures précises: présentation du compte, certifications pour les marchés, vérification des justificatifs, arrêts, inventaire, livres des mémoriaux... Ils doivent suivre avec «diligence» les recommandations, touchant aussi bien les supports que les pratiques d'écriture. Les comptes sont transcrits généralement sur parchemin, la justification des pages réservant de larges marges pour les sommes (marge droite), les ajouts et les opérations de contrôle (marge gauche). Ces registres de comptes et toutes les pièces liées transmettent une réalité économique souhaitée et définie par le prince et sa chambre des comptes. Toutefois, cette standardisation croissante ne gomme pas complètement la part d'empirisme qui préside à la réalisation des comptes, les receveurs adaptant leurs techniques et leurs outils aux besoins du terrain (fig. 4).

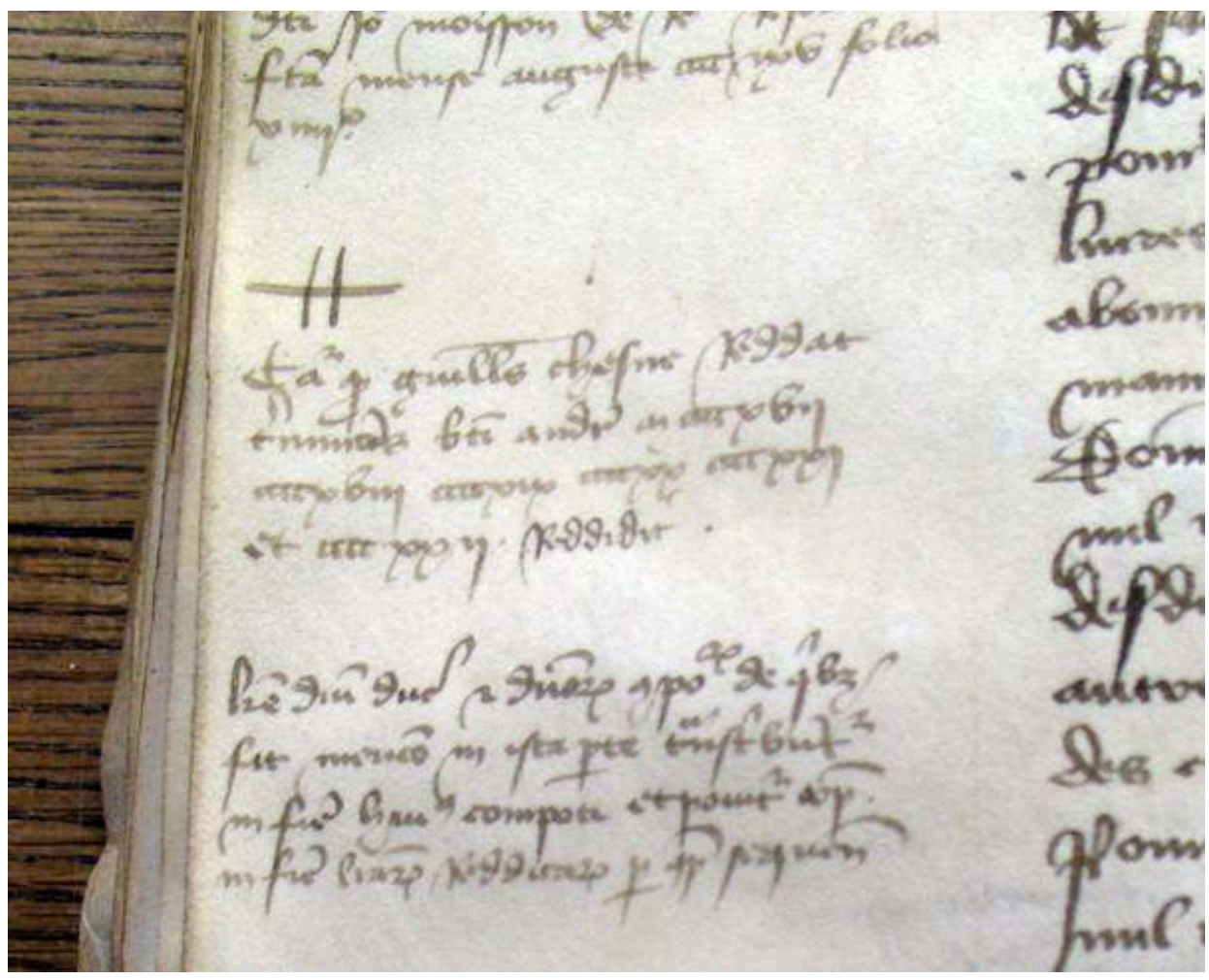

Fig. 4 - Les signes du contrôle de la chambre des comptes : compte de Bracon, 1422-1423, archives départementales de la Côte-d'Or, B3360. 


\section{Actes cisterciens et prémontrés}

9 Avec la multiplication, ces dernières années, des études s'intéressant aux riches productions scripturaires des "nouveaux monastères" créés au XII siècle, nous pouvons espérer une meilleure appréhension de ce qui pourrait s'avérer être, aussi, une forme de mise en ordre par l'écrit, articulant à des degrés variés pragmatisme et normalisation. Les trois fonds diplomatiques, un prémontré et deux cisterciens, présentés - Dilo, Vauluisant, La Bussière -, en cours d'étude, ont permis de poser déjà quelques questions.

10 Fondée en 1132, dans le sud du Sénonais, au cœur de la forêt d'Othe, et très vite devenue prémontrée, l'abbaye de Dilo - dép. Yonne, arr. Sens, cant. Cerisiers, com. Arces-Dilo -, s'implante à proximité de trois abbayes cisterciennes, Vauluisant au nord, Les Écharlis au sud-ouest et Pontigny au sud. Ses archives, après avoir été transférées au XVIII ${ }^{e}$ siècle à Saint-Florentin, sont conservées actuellement en grande partie aux archives départementales de l'Yonne, ainsi qu'à la bibliothèque municipale de Joigny, pour quelques pièces. Un ensemble de 167 actes, dont une centaine d'originaux, sont conservés pour le seul $\mathrm{xII}^{\mathrm{e}}$ siècle. Jean-Luc Benoit suggère que ces actes ont probablement été réunis dans un cartulaire aujourd'hui perdu, moderne peut-être. Ce cartulaire est connu grâce à des diverses mentions : des copies d'actes le décrivent comme "très ancien", "recouvert d'ais de bois"; un petit recueil de la fin du $\mathrm{XVIII}^{\mathrm{e}}$ siècle, réalisé par un chanoine, en serait la copie. Ce registre regroupe en tête les diplômes et les bulles, suivis des actes mis en ordre chronologique jusqu'en 1204 . Employant parfois des formules propres, inusitées ailleurs, rendant compte des échanges entretenus avec la société environnante, les chartes se rapportent également à l'entrée de femmes dans la dépendance féminine, située plus au nord dans la vallée de la Vanne (12 actes), et gardent la trace de l'intervention de Thomas Becket en faveur de Dilo, lors de son séjour dans la région en 1166-1170. Certains actes ont été grattés et trois sont des faux réalisés à la fin $\mathrm{du} \mathrm{xv}^{\mathrm{e}}$-début $\mathrm{XvI}^{\mathrm{e}}$ siècle. Les actes émanent majoritairement des évêques ( $65 \%$ ), dont $47 \%$ des archevêques de Sens. L'importance de la chancellerie archiépiscopale peut être mesurée par la présence, dans les actes de différents établissements dont Pontigny, d'une même main et de formules peu fréquentes, qui reviennent à Fromond, notaire et chapelain de l'archevêque (main $F$ ), ainsi que d'actes "décorés " datant de l'archiépiscopat de Guillaume aux Blanches Mains - archevêque de Sens entre 1164 et 1179.

11 Les archevêques de Sens sont également très présents dans les actes de l'abbaye cistercienne de Vauluisant, située à une trentaine de kilomètres au nord de Dilo, à proximité de Courgenay - dép. Yonne, arr. Sens, com. Villeneuve-l'Archevêque. Fondée en 1127, la documentation diplomatique de cette fille de Preuilly commence à être étudiée de façon exhaustive par Marlène Hélias-Baron, alors qu'on attend prochainement la publication de l'enquête sur la bibliothèque de l'abbaye par François Bougard et Pierre Petitmengin ${ }^{16}$. En plus des très nombreux actes originaux - 130 ont été répertoriés - et de plusieurs chirographes, conservés aux archives départementales de l'Yonne et de l'Aube, on dispose également, pour Vauluisant, d'un cartulaire des XII ${ }^{\mathrm{e}}$ XIII ${ }^{e}$ siècles (PARIS, BnF, lat. 9901), édité par William Duba en $1996{ }^{17}$, et d'un inventaire ${ }^{18}$. À l'inverse d'autres maisons cisterciennes, aucune pancarte ne semble avoir été réalisée; la série de courtes notices, concernant essentiellement des donations in elemosinam de biens puis de droits, connues par le cartulaire, a peut-être été préférée 
comme forme d'enregistrement. Au départ de cette enquête, trois points ont retenu l'attention: la notice de fondation, la bonne conservation des actes originaux et le noyau du XII ${ }^{e}$ siècle du cartulaire. La notice de fondation de Vauluisant a été transcrite par la première main du cartulaire au XII ${ }^{\mathrm{e}}$ siècle, précédée du titre rubriqué Exordium fundamenti sancte Marie Vallis Lucentis. Le terme " exorde » n'est pas sans rappeler les premiers récits des origines de Cîteaux ${ }^{19}$ : utilisé spécialement par le cartulariste concepteur, il confère à ce texte une valeur plus narrative que diplomatique. Comme celles de Pontigny, Preuilly et Reigny, la notice de fondation de Vauluisant semble avoir été composée plusieurs années après la fondation, après la mort des fondateurs, dans un but mémoriel évident, mais aussi pour assurer son rang à l'intérieur de l'ordre cistercien. Le cartulaire réalisé en 1164, selon François Bougard, à la suite du privilège pontifical de 1163 , et remanié au XIII ${ }^{\mathrm{e}}$ siècle, est un grand volume de 107 feuillets contenant 420 chartes - délivrées entre 1127 et 1229 -, dont 244 pour le seul XII ${ }^{\mathrm{e}}$ siècle. Les caractéristiques globales de ce fonds ressemblent beaucoup à celles du fonds de Reigny, avec un faible nombre de documents aux origines du monastère, suivi d'une augmentation régulière au cours $d u \mathrm{XII}^{\mathrm{e}}$ siècle, puis significative au XIII ${ }^{\mathrm{e}}$ siècle. Si les actes émanent principalement des archevêques de Sens, à partir de 1200 des officiaux (magistri) interviennent à la place des prélats de Sens (dès 1202), d'Auxerre et de Meaux.

Le nombre important des chartes épiscopales provenant des évêques de Langres, d'Autun et des chirographes, ainsi que l'absence de pancartes, caractérisent aussi le fonds de l'abbaye cistercienne de La Bussière - dép. Côte-d'Or, arr. Beaune, com. Blignysur-Ouche -, située à une quarantaine de kilomètres de l'abbaye de Cîteaux. L'édition de ces chartes est en préparation par Mickaël Lauquin et Nicolas Perreaux, étudiants de l'université de Bourgogne, à la suite et parallèlement à leurs travaux de master, pour l'un, et de doctorat, pour l'autre. Conservé aux archives départementales de la Côted'Or, essentiellement sous les côtes 12 H 1-220, le fonds de La Bussière est riche de plus de 200 originaux, pour la période allant de la fondation de l'abbaye en 1131 jusqu'à 1250. D'un type traditionnel et courant chez les cisterciens, ces actes s'insèrent fréquemment dans des micro-séries, clairement organisées et formalisées, qui créent des sortes de dossiers, comme ceux relatifs à un même bien par exemple (fig. 5). 


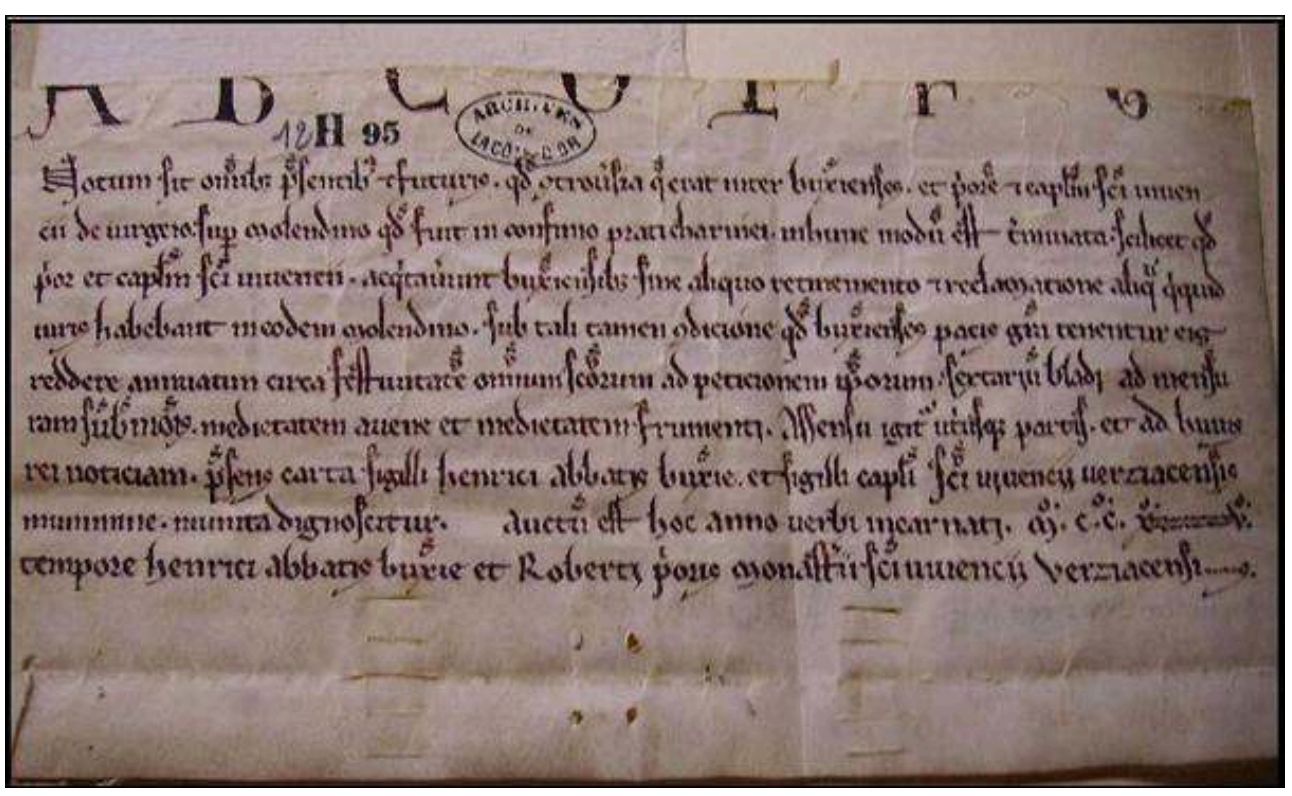

Fig. 5 - Chirographe de 1215 (archives départementales de la Côte-d'Or, 12 H 95). Résolution de conflit entre La Bussière et Saint-Vivant.

13 Sur la base de ces originaux conservés, du cartulaire - PARIS, BnF, lat. 2563 -, de copies, de vidimus et d'un terrier de 1556, les pratiques d'écriture et de conservation adoptées par les moines de La Bussière apparaissent d'une grande diversité. La subdivision du chartrier traduit bien l'implantation géographique et juridique du domaine dans une zone seigneuriale peu éloignée de l'abbaye, l'Auxois. Les mentions dorsales permettent d'identifier dix layettes de rangement des chartes et donc de localiser ce domaine: titulus castri novi (Châteauneuf), de burre (Beurey), de charne (Charny), de comarre (Commarin), de eschannay (Échannay), de marrigne (Marigny), de poule (Pouilly), de sombernon, de vouchier (Veuchey) et vietelli (Vitteaux).

14 La mise en contexte quantitative, chronologique et textuelle des actes de La Bussière, avec seize autres ensembles cisterciens, soumise à des analyses factorielles, permet de dresser le périmètre dans lequel l'abbaye s'insère, tout en posant les bases et les questions d'une approche, effectivement comparée, de la production scripturaire de l'ordre cistercien. On peut ainsi constater que La Bussière se place près de Clairvaux, Cheminon, Fontenay, Bonnevaux et à proximité des étiquettes chronologiques 1181-1190 et 1191-1200. À l'opposé, proche de 1131-1140 et surtout de 1141-1150, on observe un autre groupe formé par Pontigny, Bellevaux, Preuilly et Auberive. Pour vérifier l'existence des similitudes dans les rythmes de production documentaire entre ces deux groupes et tenter d'amorcer une hypothèse, une série de tests a été effectuée à partir des données des tableaux de décompte des actes de vingt maisons, dressés par Constance Bouchard dans Holy Entrepreneurs. Le premier groupe, celui de La Bussière et de Cîteaux, dispose d'un excédent documentaire pour la fin et une faiblesse pour le début du XII ${ }^{e}$ siècle. Le deuxième groupe, celui de Pontigny et Bellevaux, connaît une situation inverse. Par ailleurs, en utilisant le coefficient de corrélation linéaire pour analyser toutes les décennies, on observe, autour de 1160, un tournant important en matière de production documentaire à l'échelle de l'ensemble des établissements analysés, qu'il faudrait encore expliquer. Quant aux tests sur le vocabulaire, ils montrent que la proximité géographique est un facteur non négligeable à prendre en compte dans la diffusion des termes et des formules. La Bussière présente, ainsi, un 
lexique plutôt proche de celui de Mores, mais aussi de ceux d'Autun et de Langres, dont les évêques ont joué un rôle important dans la constitution du patrimoine de l'abbaye (fig. 6, 7 et 8).

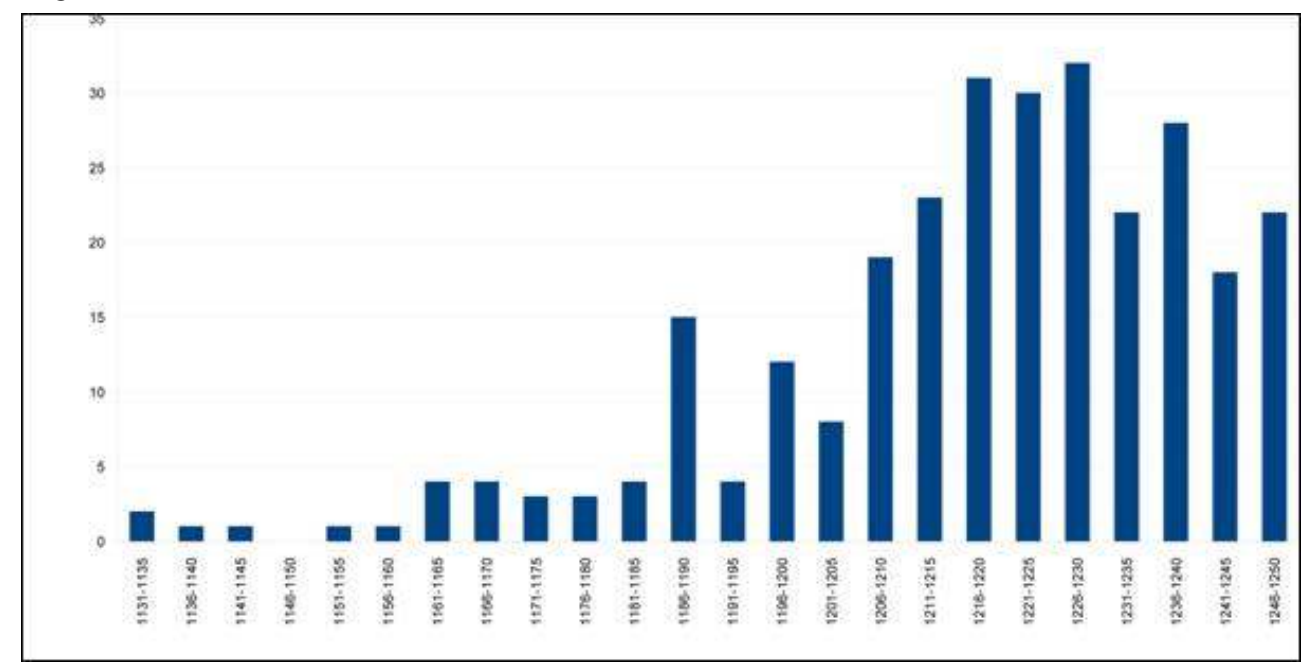

Fig. 6 - Un accroissement fortement inégalitaire.

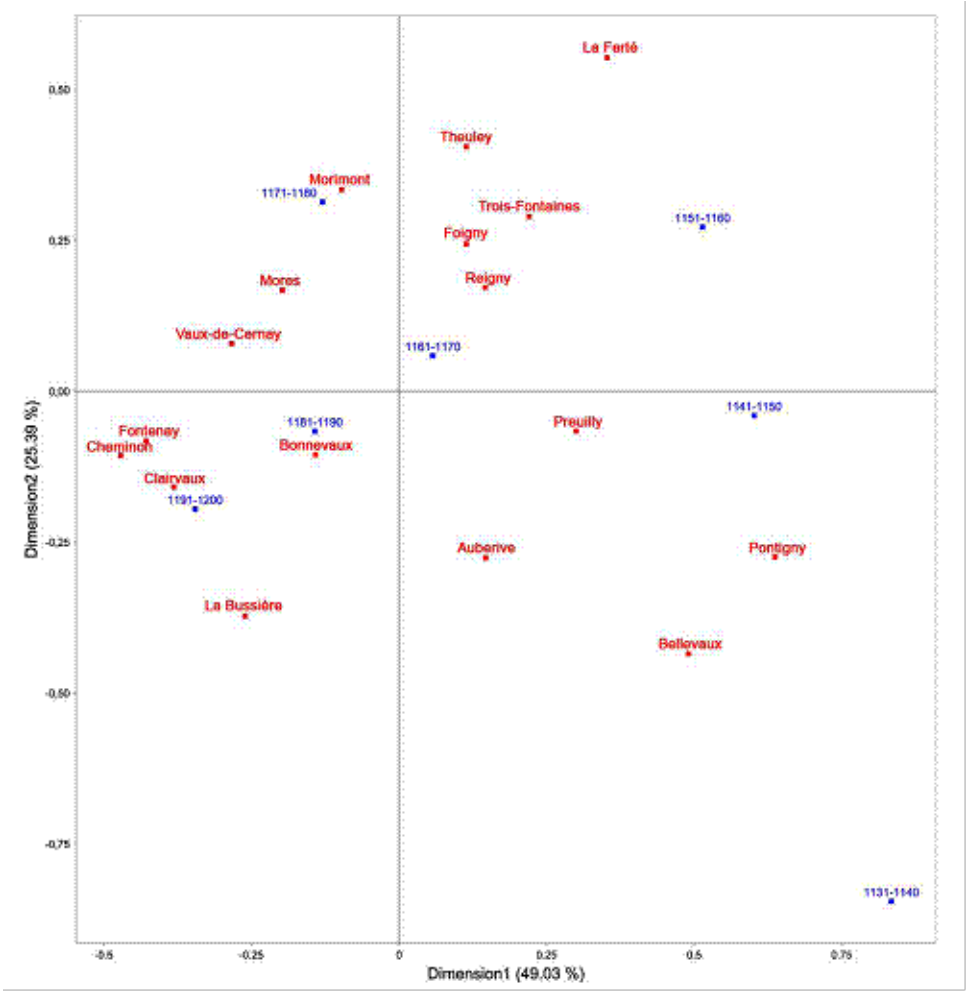

Fig. 7 - Analyse factorielle [AFC réalisée avec $\mathrm{R}$ - packages : FactoMineR; dynGraph].

\begin{tabular}{|c|c|c|c|c|c|c|c|}
\hline & $1131-1140$ & 1141.1150 & $1151-1160$ & $1161-1170$ & $1171-1180$ & 1181.1190 & $1191-1200$ \\
\hline 1131.1140 & 1 & 0.73 & 0.32 & 0.17 & 0.02 & 0.09 & 0.02 \\
\hline $1141-1150$ & 0.73 & 1 & 0.56 & 0.34 & 0.07 & 0.21 & 0.05 \\
\hline $1151-1160$ & 0.32 & 0.66 & 1 & 0.19 & 0.07 & 0.05 & 0 \\
\hline $1161-1170$ & 0.17 & 0.34 & 0.19 & 1 & 0.48 & 684 & 0.8 \\
\hline $1171-1180$ & 0.02 & 0.07 & 0.07 & 0.48 & 1 & 0.54 & as \\
\hline $1181-1190$ & 0.09 & 0.21 & 0.05 & 059 & 054 & 1 & 08 \\
\hline $1191-1200$ & 0.02 & 0.05 & 0 & D.68 & 0.51 & 06 & 1 \\
\hline
\end{tabular}

Fig. 8 - Coefficients de corrélation linéaire. La question des liens entre décennies (production documentaire). 
Ces méthodes d'analyse prometteuses, la continuité dans les réalisations, les enrichissements documentaires et techniques, ainsi que le soutien financier renouvelé de la Région Bourgogne à notre projet, nous rassureNT sur l'avenir et nous conforteNT dans les choix faits et les principes adoptés dans le programme des CBMA.

Rappel du programme

- Eliana MAGNANI et Marie-José GASSE-GRANDJEAN, Le programme CBMA : bilan 2010

- Johan AHLFELDT, The database Regnum Francorum Online (RFO). Towards a semantic web

- Sylvie BEPOIX, Codicologie et organisation des comptabilités bourguignonnes, fin XIVe-XVe siècle, un système au service du prince

- Jean-Luc BENOIT, Recherches sur les actes de l'abbaye prémontrée de Dilo (Yonne, diocèse de Sens)

- Marlène HÉLIAS-BARON, Le cartulaire de l'abbaye de Vauluisant (Yonne, diocèse de Sens)

- Nicolas PERREAUX et Mickaël LAUQUin, Les actes de l'abbaye de La Bussière (Côte-d'Or, diocèse d'Autun)

\section{Liens utiles}

- Projet CBMA sur le site web Artehis : http://www.artehis-cnrs.fr/CBMA-Chartae-BurgundiaeMedii-Aevi

- Documentation CBMA : http://www.artehis-cnrs.fr/page- documentaire-CBMA

- Serveur CBMA sous Philologic : http://www.artehis- cbma.eu/

- Application SIG : http://phalombe.u-bourgogne.fr/cbma/

- Wiki « Chartes bourguignonnes » : http://fr.wikipedia.org/wiki/Chartes_bourguignonnes

- Blog Laetus diaconus : http://laetusdiaconus.hypotheses.org/

\section{NOTES}

1. Ce serveur est accessible à partir de la page web « Projet CBMA » : http://www.artehis-cnrs.fr/ La-base-de-donnees-CBMA\%20

2. Notons qu'une aide est disponible en ligne et que cette application fonctionne de façon optimale avec Firefox.

3. Cette couche "diocèse avant 1317 » a été ajoutée grâce au travail de cartographie et à l'autorisation de Thomas Aréal, étudiant de Clermont 2, d'après les cartes des diocèses établies par dom Dubois et publiées dans la Revue des Annales : http://georezo.net/forum/viewtopic.php? $\mathrm{id}=65136 \& \mathrm{hl}=$ dioc\%E8se.

4. L. S Touff (éd.), Les comtes de Bourgogne et leurs villes domaniales. Étude sur le régime communal, forme de l'exploitation seigneuriale d'après le cartulaire de la ville d'Arbois, suivie du texte de ce Cartulaire, de pièces annexes, de notes et de table. XIII ${ }^{\mathrm{e}}$, XIV ${ }^{\mathrm{e}}$ siècles, Paris, 1899.

5. B. GASPARD, Histoire de Gigny, au département du Jura, de sa noble et royale abbaye, et de Saint-Taurin, son patron, suivie de pièces justificatives, Lons-le-Saunier, 1843.

6. J. RICHARD, Le Cartulaire de Marcigny-sur-Loire (1045-1144). Essai de reconstitution d'un manuscrit disparu, Dijon, 1957 (Analecta burgundica). Ce dernier corpus a été enregistré et mis en ligne grâce à l'aimable autorisation de l'auteur, que nous tenons à remercier ici.

7. Cartulaire de l'abbaye de Cîteaux (xIII ${ }^{\mathrm{e}}$ siècle) : AD 21, $11 \mathrm{H} 66$ (cart. 167 et 168), 1178 actes (1044-1302). 
8. Cartulaire général de Cîteaux, dit de Jean de Cirey ( $\mathrm{XV}^{\mathrm{e}}$-XVII ${ }^{\mathrm{e}}$ siècles) : AD 21, $11 \mathrm{H} 75$ (cart. 190), 315 folios, $6^{\mathrm{e}}$ volume d'un cartulaire en 9 volumes qui compte 2986 actes (1098-1609). Sur ce cartulaire voir C. REY, «L'entreprise archivistique de Jean de Cirey, abbé de Cîteaux (1476-1501). Le dossier documentaire de la seigneurie de Villars en Côte-d'Or », Bucema, 14 (2010), p. 347-351 [http://cem.revues.org/index11638.html].

9. Cartulaire du prieuré de Saint-Vivant-sous-Vergy, dépendant de l'abbaye de Cluny (xIV siècle) : AD 21, 21 H (cart. 231, 40 folios, 98 actes, 1200-1330).

10. Ce projet a fait l'objet d'un atelier le 27 janvier 2011 à l'UMR 5594 Artehis.

11. Voir les réflexions à l'issue de la rencontre: D. Russo, «Productions, remplois, mises en registre : la pratique sociale de l'écrit à travers la documentation médiévale bourguignonne ", Bucema, 14 (2010), p. 157-167 [http://cem.revues.org/index11516.html].

12. Voir le détail du projet Cantus burgundiensis dans ce bulletin.

13. En ligne : http://irhis.recherche.univ-lille3.fr/00-Comptabilites/Index-1.html.

14. Voir le compte rendu de cette rencontre dans ce bulletin. Pour l'ensemble du projet, cf. P. BECK, «Le vocabulaire et la rhétorique des comptabilités médiévales. Modèles, innovations, formalisation », Bucema, 14 (2010), p. 267-271 [http://cem.revues.org/index11578.html].

15. Voir la notice sur ces recherches dans ce numéro.

16. F. B OUgard et P. P etitmengin, avec la collaboration de J.-L. Benoît, F. DolbeAU, S. GioAnNi, C. NAUD $\dagger$, M. ROYO, P. STIRNEMANN, M. TARPINIAN et A. VAILLANT, La bibliothèque de l'abbaye cistercienne de Vauluisant. Histoire et inventaires, à paraître dans la collection «Documents, études, répertoires » (D.E.R.) de l'IRHT.

17. W. O. DUBA, The cartulary of Vauluisant. A critical edition, University of Iowa, 1994-1996.

18. PARIS, BnF, lat. 9901.

19. C. WADDELL, Narrative and legislative Texts from early Cîteaux, Cîteaux, 1999, Exordium Cistercii, cap. II, p. 180 et Exordium parvum, cap. I, p. 235.

\section{INDEX}

Mots-clés : CBMA 